ARTICLE

\title{
Structural and functional analysis of target recognition by the lymphocyte adaptor protein LNK
}

\author{
Rhiannon Morris (10) 1,2, Yaoyuan Zhang 3,4, Julia I. Ellyard (1) 3,4, Carola G. Vinuesa (1) 3,4, James M. Murphy (1) 1,2,

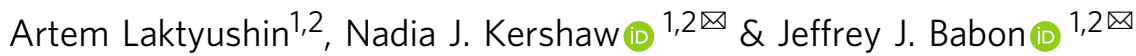

The SH2B family of adaptor proteins, $\mathrm{SH} 2-\mathrm{B}, \mathrm{APS}$, and LNK are key modulators of cellular signalling pathways. Whilst SH2-B and APS have been partially structurally and biochemically characterised, to date there has been no such characterisation of LNK. Here we present two crystal structures of the LNK substrate recognition domain, the $\mathrm{SH} 2$ domain, bound to phosphorylated motifs from JAK2 and EPOR, and biochemically define the basis for target recognition. The LNK SH2 domain adopts a canonical SH2 domain fold with an additional $\mathrm{N}$-terminal helix. Targeted analysis of binding to phosphosites in signalling pathways indicated that specificity is conferred by amino acids one- and three-residues downstream of the phosphotyrosine. Several mutations in LNK showed impaired target binding in vitro and a reduced ability to inhibit signalling, allowing an understanding of the molecular basis of LNK dysfunction in variants identified in patients with myeloproliferative disease.

\footnotetext{
${ }^{1}$ Walter and Eliza Hall Institute of Medical Research, 1G Royal Parade, Parkville, VIC 3052, Australia. ${ }^{2}$ Department of Medical Biology, The University of Melbourne, Royal Parade, Parkville, VIC 3052, Australia. ${ }^{3}$ Australia Department of Immunology and Infectious Diseases, Australian National University, Canberra, ACT, Australia. ${ }^{4}$ Australia Centre for Personalised Immunology, John Curtin School of Medical Research, Australian National University, Canberra,

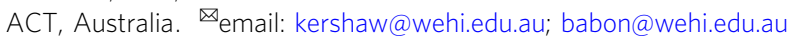


T hrombopoietin (TPO) and erythropoietin (EPO) are hematopoietic cytokines that bind specific receptors on the surface of target cells and induce activation of the Janus kinase (JAK)-signal transducer and activation of transcription (STAT) pathway. TPO is an essential modulator of megakaryopoiesis, platelet production and stem cell quiescence, whereas EPO is required for the regulation of erythropoiesis. The Lymphocyte adaptor protein ( $\mathrm{LNK}^{1}$ (also SH2B3)) is a member of the $\mathrm{SH} 2$ domain containing adaptor family of proteins, which also comprises APS (SH2B2) and $\mathrm{SH} 2 \mathrm{~B}(\mathrm{SH} 2 \mathrm{~B} 1)^{2,3}$ and negatively regulates both EPO and TPO signalling ${ }^{4,5}$ via its interaction with JAK $2^{6}$. All three proteins contain a dimerisation domain, a pleckstrin homology $(\mathrm{PH})$ domain and a Src homology 2 (SH2) domain and are involved in the regulation of various signalling pathways downstream of cytokines and growth factors $4,5,7-10$.

LNK is highly expressed in haematopoietic stem cells (HSCs), and deletion of LNK from HSCs leads to an increase in cell number and proliferative capacity ${ }^{6}$, suggesting a role for LNK in regulating HSC self-renewal. Comparably, overexpression of LNK in haematopoietic progenitor cell lines restrains TPO-induced cellular proliferation, and overexpression of LNK in primary hematopoietic cells inhibits megakaryopoiesis ${ }^{4}$. These findings are recapitulated in vivo with LNK-deficient mice displaying increased numbers of megakaryocytes that have enhanced TPO sensitivity ${ }^{4}$ as well as enhanced numbers of platelets, lymphocytes and erythroid cells $s^{3,5}$. Similarly, erythroid colony-forming progenitors from the spleens of LNK deficient mice displayed increased sensitivity to EPO stimulation ${ }^{5}$.

Given the ability of LNK to negatively regulate EPO and TPO signalling, it is unsurprising that LNK loss-of-function mutations have been identified as drivers of human myeloproliferative disease $^{11}$. In addition, there is an increased incidence of LNK mutations in leukaemic transformation of myeloproliferative neoplasms, suggesting LNK function may effect the severity of disease ${ }^{12-14}$. The PH and SH2 domains of LNK are both hotspots for mutations ${ }^{15}$, and are essential for LNK function. Currently the mechanism by which LNK negatively regulates signalling is poorly understood, and so the role of LNK substitutions in the onset and progression of disease is uncertain. Understanding how the LNK SH2 domain interacts with substrates may shed light on how negative regulation of various signalling molecules occurs, and why mutations in the LNK SH2 domain contribute to disease burden in patients.

LNK has also been suggested to bind a suite of other signalling proteins involved in haematopoiesis, proliferation and differ-

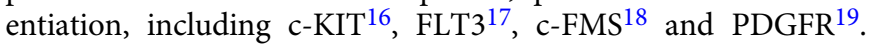
The need to establish which signalling proteins are regulated by LNK is underscored by the identification of LNK mutations in patients with a range of inflammatory, immune and haematopoietic diseases including cancer ${ }^{20-25}$. Characterisation of the substrate recognition domain of LNK, the $\mathrm{SH} 2$ domain, would therefore be a step towards a comprehensive overview of the proteins and pathways that LNK negatively regulates. While the structural details of APS and SH2-B have been elegantly characterised by the Hubbard laboratory ${ }^{2,26,27}$, to date there have been no structural or biochemical studies of LNK, with only a single published example of the successful expression of any part of the LNK protein ${ }^{28}$ and no example of the yields and purity required for an in-depth structural and biochemical analysis.

Here we present the structure of the substrate recognition domain of LNK, the SH2 domain, in complex with phosphopeptide motifs from JAK2 (pY813) and EPOR (pY454) and biochemically characterise these interactions. The LNK SH2 domain, unlike APS but similarly to SH2-B, adopts a monomeric structure and binds its target sequences in a canonical linear, extended conformation. We tested the ability of the LNK SH2 domain to bind to a number of different phosphorylated peptides from proteins proposed to be regulated by LNK, revealing the highest affinities were for sites on JAK2, JAK3 and EPOR. Studies of $\mathrm{SH} 2$ domain point mutations identified in patients with myeloproliferative neoplasms revealed that the $\mathrm{SH} 2$ domain displayed a decrease in affinity for ligands and the same mutations, when incorporated into the full-length protein had a reduced capacity to regulate signalling in vitro. Together these findings detail the specificity of the LNK SH2 domain and aid our understanding of how single point mutations affects LNK function, contributing to haematological diseases.

\section{Results}

Crystal structure of the LNK SH2 domain in complex with the JAK2 pY813 motif. We attempted a number of different bacterial and baculovirus expression systems to produce active $\mathrm{SH} 2$ domain from both human and mouse LNK including. Whilst GST and His6-based fusion systems were unsuccessful, a NusA fusion of the M. musculus LNK SH2 domain (using the domain boundaries from Machida et $\mathrm{al}^{28}$ ) yielded folded, monomeric protein once the fusion tag was removed as determined by size exclusion chromatography and thermal shift assay. The LNK SH2 domain was then co-crystallised with a 12-mer phosphopeptide corresponding to JAK2 pY813 and flanking residues (designated below as $+1,+2 \ldots$ and $-1,-2 \ldots$ relative to $\mathrm{pTyr}$ ). Importantly, the murine LNK SH2 domain shares $92.9 \%$ identity with the H. sapiens orthologue (Supplementary Fig. 1), having high conservation across all phosphopeptide-binding residues, with only a threonine to serine substitution from human to mouse within the phosphotyrosine binding pocket. The crystal structure of this complex was solved to $1.9 \AA$ resolution (Supplementary Table 1), revealing that the $\mathrm{LNK} \mathrm{SH} 2$ domain adopts a typical $\mathrm{SH} 2$ domain fold comprising three central $\beta$-strands flanked by two $\alpha$-helices. In addition, there is a short helix similar to that found in the SOCS and STAT proteins located just outside the SH2 domain boundary on the N-terminal side $\mathrm{e}^{29,30}$ (Fig. 1a and 1b). This helix extends behind the central $\beta$ sheet and is positioned over a hydrophobic patch. A leucine in this helix (Leu330) begins an LSxYP motif conserved across all three SH2B family members and inserts its sidechain into a hydrophobic pocket on this surface (Fig. 1b and Supplementary Fig. 2). Thus, this N-terminal helix may be a conserved feature across the SH2B family. Additionally, this helix appears to be conserved across several vertebrate species (Supplementary Fig. 2), suggesting this N-terminal helix may be an evolutionarily conserved feature in LNK. Another unexpected feature is a disulfide bond formed between Cys 421 and Cys 425 of the BG loop, a feature not present in $\mathrm{SH} 2 \mathrm{~B}$ or APS; whether this would be present in vivo is unclear.

The pTyr of the JAK2 pY813 phosphopeptide inserts into the canonical phosphotyrosine binding pocket of the LNK SH2 domain, formed by Arg 343, Arg 364, Ser 366, Arg 369, His 385 and Arg 387. The phosphate moiety of pY813 forms hydrogen bonds with the invariant Arg 364 and the highly conserved Arg 343 along with the backbone amide nitrogen of Glu 367, and the sidechain of Ser 368 (Fig. 1c, d). Glu 814 of the JAK2 peptide $(\mathrm{P}+1)$ appears to be a key determinant of binding as it forms a hydrogen bond with Lys 384 of LNK. In addition, Leu $816(\mathrm{P}+3)$ inserts into a hydrophobic pocket common in SH2 domains that is formed by Leu 386, Val 398, Leu 401, Phe 403, Phe 413, Ile 418 and Leu 420 of LNK (Fig. 1c). Numerous backbone interactions form the remainder of intermolecular contacts (Fig. 1b and Supplementary Fig. 3). 
a
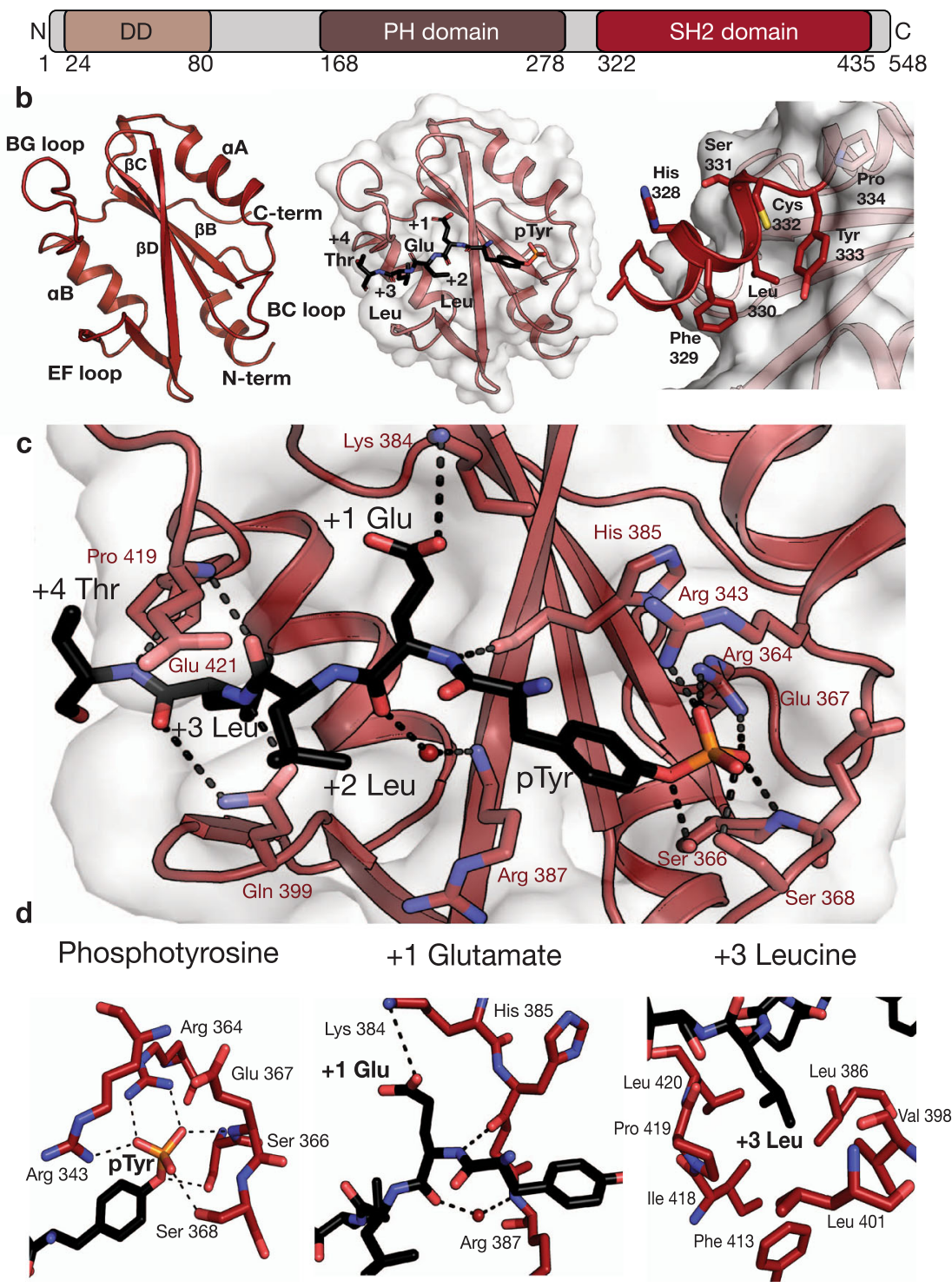

Fig. 1 The LNK SH2/JAK2 pY813 co-crystal structure. a Schematic representation of the LNK domain architecture. LNK comprises an N-terminal dimerisation domain, a central plekstrin homology domain and a C-terminal SH2 domain. $\mathbf{b}$ (left) Cartoon representation of the backbone of the WT LNK $\mathrm{SH} 2$ domain with the secondary structural features indicated (peptide not shown), (middle) LNK SH2 domain structure with the JAK2 pY813 peptide shown, and (right) the LNK SH2 domain N-terminal helix extending behind the central $\beta$ strands, positioned over a hydrophobic surface on the $\mathrm{SH} 2$ domain. c Interactions between the LNK SH2 domain (red) and JAK2 pY813 peptide (black). The phosphotyrosine occupies the phosphotyrosine binding pocket of the SH2 domain and is coordinated by R364, S368, R343, and the nitrogen of E367 of LNK. The +1 Glu forms a salt bridge with K384 of LNK and the +3 Leu sits in a hydrophobic pocket formed in part by the EF and BG loops of LNK. d Specific interactions between the LNK SH2 domain and the phosphotyrosine, $+1 \mathrm{Glu}$, and +3 Leu residues highlighted.

Binding of the LNK SH2 domain to phosphotyrosine motifs resembles the binding mode of $\mathrm{SH} 2 \mathrm{~B}$ and is distinct from that of APS. The SH2 domains of APS and LNK share 64.6\% sequence identity (Fig. 2a), with high conservation around the phosphotyrosine binding pocket (Supplementary Fig. 4a) however the structures are notably different. APS forms a dimer (PDB ID: 1 RQQ $)^{26}$ whereas LNK is monomeric. APS dimerisation occurs via its $\alpha \mathrm{B}$ helix, which is longer and extends behind the $\mathrm{SH} 2$ domain (Fig. 2b). The +3 hydrophobic pocket forms part of this dimer interface and as such peptide binding is considerably altered (Supplementary Fig. 4a) and differs from most SH2 domains by not adopting a linear, extended conformation.

In contrast, LNK is structurally more similar $\mathrm{SH} 2 \mathrm{~B}$, which also binds JAK2 pY813. The SH2 domains of LNK and SH2B share
$68.7 \%$ sequence identity (Fig. 2a) and the crystal structures of their SH2 domains (SH2B PDB ID: $2 \mathrm{HDX})^{2}$ align with a rootmean-square deviation (RMSD) of $1.26 \AA$ over 106 atoms (Fig. 2b). There are subtle differences in pTyr binding between these two family members despite a high degree of conservation across the peptide binding residues (Supplementary Fig. 4b). In both, the phosphate group of pY813 occupies the canonical phosphotyrosine binding pocket and is coordinated by an invariant arginine (Arg 364 in LNK), however in LNK, Arg 369 and 387 are involved in a hydrogen bonding network with Glu 372 , and do not interact with the phosphate moiety. In contrast, in $\mathrm{SH} 2 \mathrm{~B}$, the corresponding three residues form different interactions and the Arg 369 equivalent (Arg 560) forms a salt bridge with the phosphate of pTyr. LNK, like SH2B also displays 


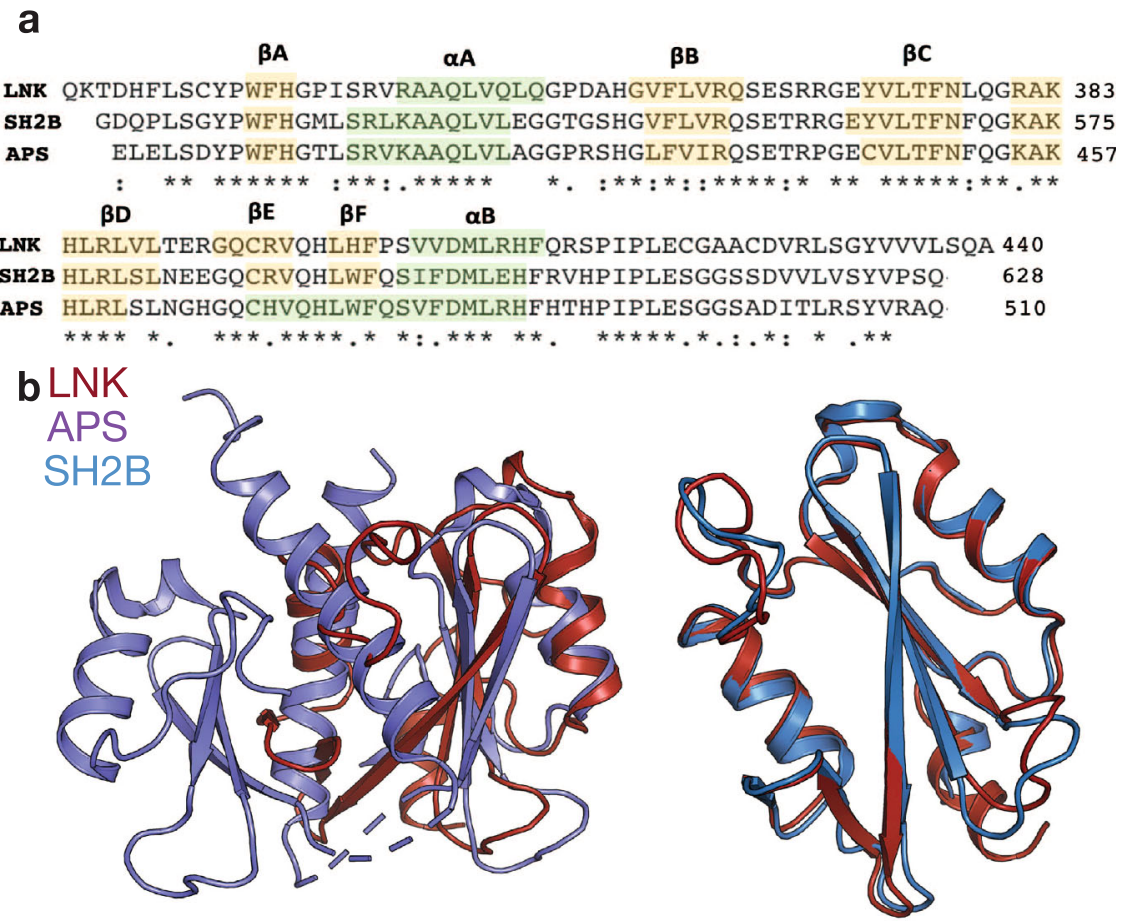

Fig. 2 Comparison of the LNK, APS and SH2B SH2 domains. a Alignment of APS, SH2B and LNK SH2 domain protein sequences, highlighting high sequence conservation between the three family members. b Alignment of the LNK and APS (PDB ID: 1RQQ) SH2 domains, with an overall RMSD of $2.4 \AA$ and alignment of LNK and SH2B (PDB ID: 2HDX) SH2 domains with an overall RMSD of $1.3 \AA$.
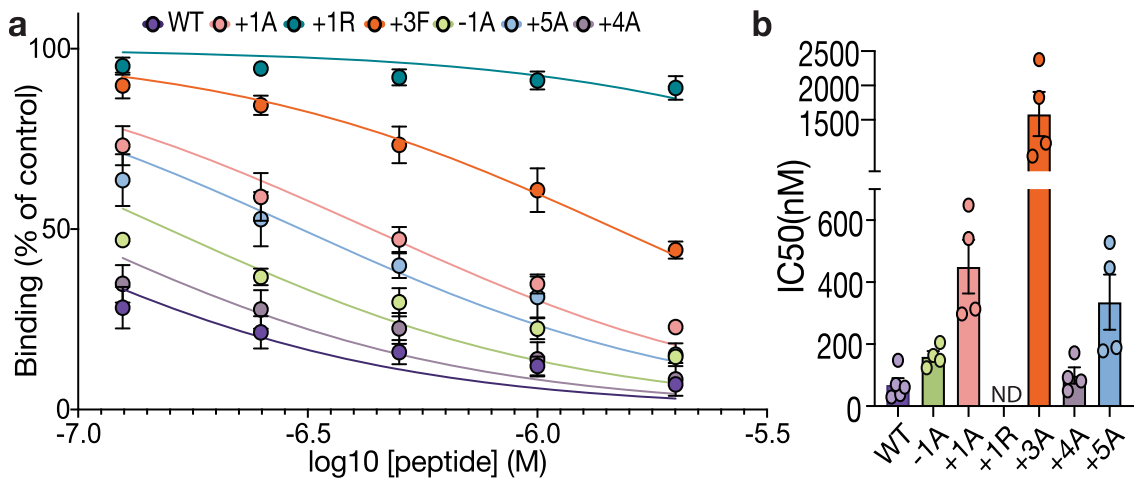

Fig. 3 Mutational Analysis of the LNK binding site in JAK2. a Percentage of binding (measured by the response at equilibrium) to the IL6ST pY757 peptide chip after pre-incubation with varying concentrations of mutant JAK pY813 peptides. b Point at which $50 \%$ of binding is inhibited shown as $\mathrm{IC}_{50}$. For both, data are shown as mean \pm SEM of technical replicates from at least two independent experiments (WT data were from five samples from $n=3$ independent experiments, all mutant data were from four samples from $n=2$ independent experiments).

specificity for a Glu at the +1 position, however in LNK this is facilitated by a salt bridge to Lys 384 , whereas in SH2B the equivalent lysine hydrogen bonds to Ser 613 which likewise hydrogen bonds to the +1 Glu.

Specificity for the LNK SH2 domain is conferred by amino acids one-residue, three-residues and five-residues downstream of the target phosphotyrosine. $\mathrm{SH} 2$ domains typically bind ligands with a specific preference for amino acids at the -2 to +5 positions, relative to phosphotyrosine ${ }^{31}$. The LNK SH2/JAK2 pY813 structure suggests that +1 and +3 residues contribute to high affinity binding. To determine the contribution of individual residues, we made a series of mutations within the JAK2 phosphopeptide, and measured their affinity for the LNK SH2 domain via a surface plasmon resonance (SPR) competition assay (Fig. 3). The LNK SH2 domain was incubated with increasing concentrations of mutant JAK2 pY813 peptides before being passed over a chip coated with an immobilised low affinity phosphopeptide derived from a site on the intracellular domain of the IL-6 receptor, IL6ST. While the $\mathrm{IC}_{50}$ of the WT pY813 peptide was 60 $\mathrm{nM}$, mutation of residues at the $+1,+3$, and to a lesser extent +5 , positions decreased the affinity of the interaction (Fig. 3). In particular, mutation of the +1 Glu to $\operatorname{Arg}(+1 \mathrm{R})$ and the +3 Leu to Phe $(+3 \mathrm{~F})$ resulted in a $>80$-fold and $\sim 25$-fold decrease in affinity respectively (Table 1 ). The +2 residue was not mutated as the structure indicates its sidechain points towards solvent. These findings, in combination with the $\mathrm{SH} 2$ domain structure, indicate three residues as key binding determinants; Glu 814, Leu 816 and Glu 818, in addition to pTyr 813 .

Identification of phosphotyrosine binding motifs in JAK3, EPOR, FLT3 and c-KIT. The LNK SH2 domain has been proposed to interact with a suite of phosphorylated sites on various signalling proteins ${ }^{16-19,32}$. Here, using an SPR competition assay, 


\section{Table 1 Peptide sequences and $\mathrm{IC}_{50}$ values from mutant JAK2 pY813 competition assay.}

\begin{tabular}{|c|c|c|c|c|c|c|c|c|}
\hline \multirow[t]{2}{*}{ Mutant } & \multicolumn{7}{|c|}{ Residues } & \multirow{2}{*}{$\begin{array}{l}I_{50}(n M) \pm \\
\text { SEM }^{a}\end{array}$} \\
\hline & -1 & pY & +1 & +2 & +3 & +4 & +5 & \\
\hline$\overline{\mathrm{WT}}$ & $D$ & pY & $E$ & $\mathrm{~L}$ & $\mathrm{~L}$ & $\mathrm{~T}$ & $E$ & $60 \pm 20$ \\
\hline$-1 \mathrm{~A}$ & A & pY & $\mathrm{E}$ & $\mathrm{L}$ & L & $\mathrm{T}$ & $\mathrm{E}$ & $150 \pm 20$ \\
\hline$+1 \mathrm{~A}$ & D & pY & A & L & L & $\mathrm{T}$ & $\mathrm{E}$ & $430 \pm 90$ \\
\hline$+1 R$ & $D$ & pY & $\mathbf{R}$ & $\mathrm{L}$ & $\mathrm{L}$ & $\mathrm{T}$ & E & $>5000$ \\
\hline$+3 \mathrm{~F}$ & D & pY & $\mathrm{E}$ & $\mathrm{L}$ & $\mathbf{F}$ & $\mathrm{T}$ & $\mathrm{E}$ & $1430 \pm 300$ \\
\hline$+4 \mathrm{~A}$ & $\mathrm{D}$ & pY & $\mathrm{E}$ & $\mathrm{L}$ & L & A & $E$ & $90 \pm 25$ \\
\hline$+5 \mathrm{~A}$ & $\mathrm{D}$ & pY & $\mathrm{E}$ & $\mathrm{L}$ & L & $\mathrm{T}$ & A & $300 \pm 90$ \\
\hline
\end{tabular}

Target phosphotyrosine and mutated residues are highlighted in bold. Data are shown as mean \pm SEM of technical replicates from at least two independent experiments (WT, five samples from $n=3$ independent experiments, all mutants, four samples from $n=2$ independent experiments).

${ }^{a} C_{50}$ as determined by SPR competition assay.

we examined binding of the LNK SH2 domain to those suggested sites from c-KIT, c-FMS, FLT3 and PDGFR and to all intracellular sites from EPOR and TPOR. In addition, binding to the activation loops of JAK2 and IRK, and pY785 of JAK3 were also examined. Although biophysical methods do not prove that an interaction exists in vivo, they provide a powerful tool to show which interactions do not occur. Given that $\mathrm{SH} 2$ domains will bind to most phosphorylated sequences to some degree, in line with the literature on $\mathrm{SH} 2$ domains and their targets ${ }^{33}$ we applied a threshold cut-off of $2000 \mathrm{nM}$, where an $\mathrm{IC}_{50}$ over this value indicates a physiologically irrelevant interaction. As shown in Table 2, we identified two high-affinity phosphotyrosine motifs from JAK3 and EPOR in addition to several moderate-affinity motifs from EPOR, FLT3 and c-KIT. These sequences all contain a hydrophobic residue in the +3 position. Peptides with an $\mathrm{IC}_{50}$ greater than $2000 \mathrm{nM}$ generally either lacked a hydrophobic residue at this position or contained an Arg at the +1 which we had previously shown abolished binding (Fig. 3). Despite previous reports implicating pTyr residues of PDGFR and c-FMS, as LNK ligands, in our hands, we did not observed binding of the LNK $\mathrm{SH} 2$ domain to phosphopeptides corresponding to these residues.

Crystal Structure of the LNK SH2 domain in complex with the EPOR pY454 motif. To understand how the EPOR pY454 peptide, which contains a leucine at the +1 position, could bind the LNK SH2 domain with high affinity, we determined the complex structure to $2.35 \AA$ resolution (Supplementary Table 1). Overall, the structure is very similar to the LNK SH2/JAK2 pY813 structure with an RMSD of $1.0 \AA$ over 111 residues $\left(\mathrm{DALI}^{34}\right)$ (Fig. $4 \mathrm{a}$ and Supplementary Fig. 5). The LNK SH2 domain accommodates the leucine of the EPOR pY454 peptide at the key +1 position in a subtly different manner to the glutamate of the JAK2 pY813 peptide. The aliphatic portions of both the +1 glutamate and leucine sit on a hydrophobic surface of LNK, however the glutamate sidechain bends so that the carboxylate faces away and forms a salt bridge with Lys 384 (Fig. 4b, c). The side chain of the EPOR pY454 +1 leucine is accommodated by a shift in the BG loop of LNK by $\sim 5 \AA$, although the exact positioning may be influenced by an adjacent crystal contact. In concordance with their similar binding modes, the surface area buried by each peptide is similar $(\sim 580 \AA)$.

Mutations found in human LNK impair target binding. Several substitutions in LNK have been linked with diseases including haematological cancers ${ }^{11,35}$, autoimmune disorders ${ }^{24,36}$ and heart disease $21,23,37$. Most of these occur within the $\mathrm{PH}$ domain however substitutions located within the $\mathrm{SH} 2$ domain have also been

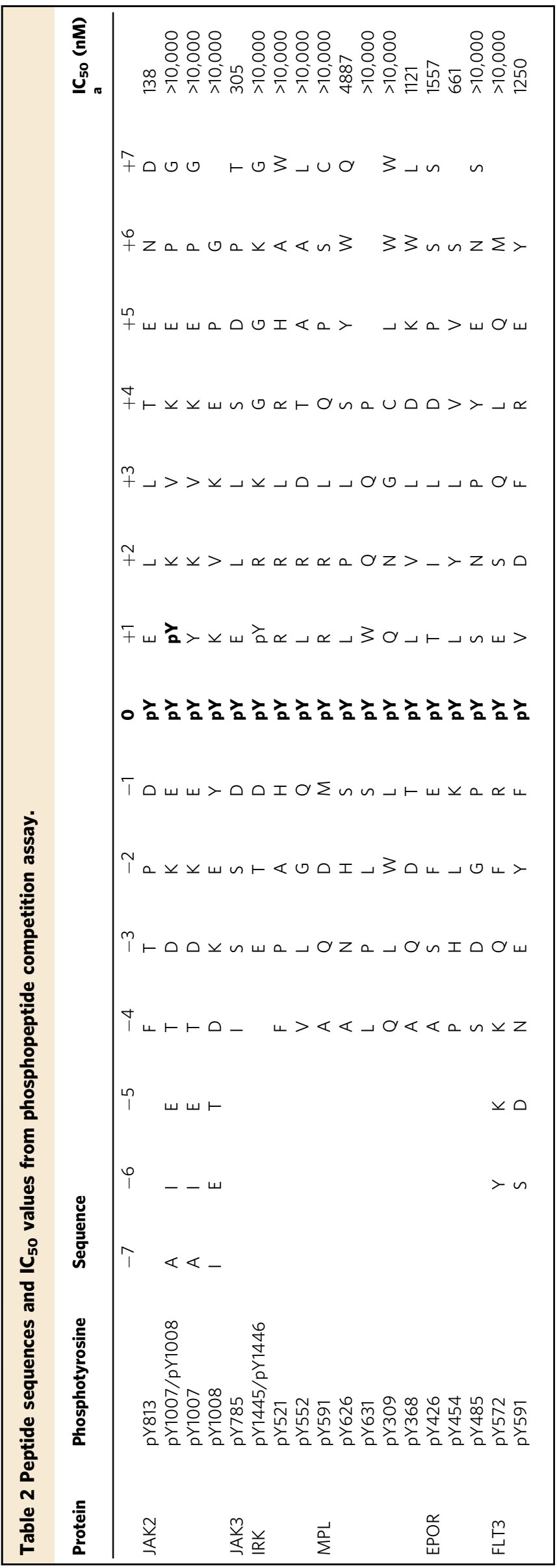




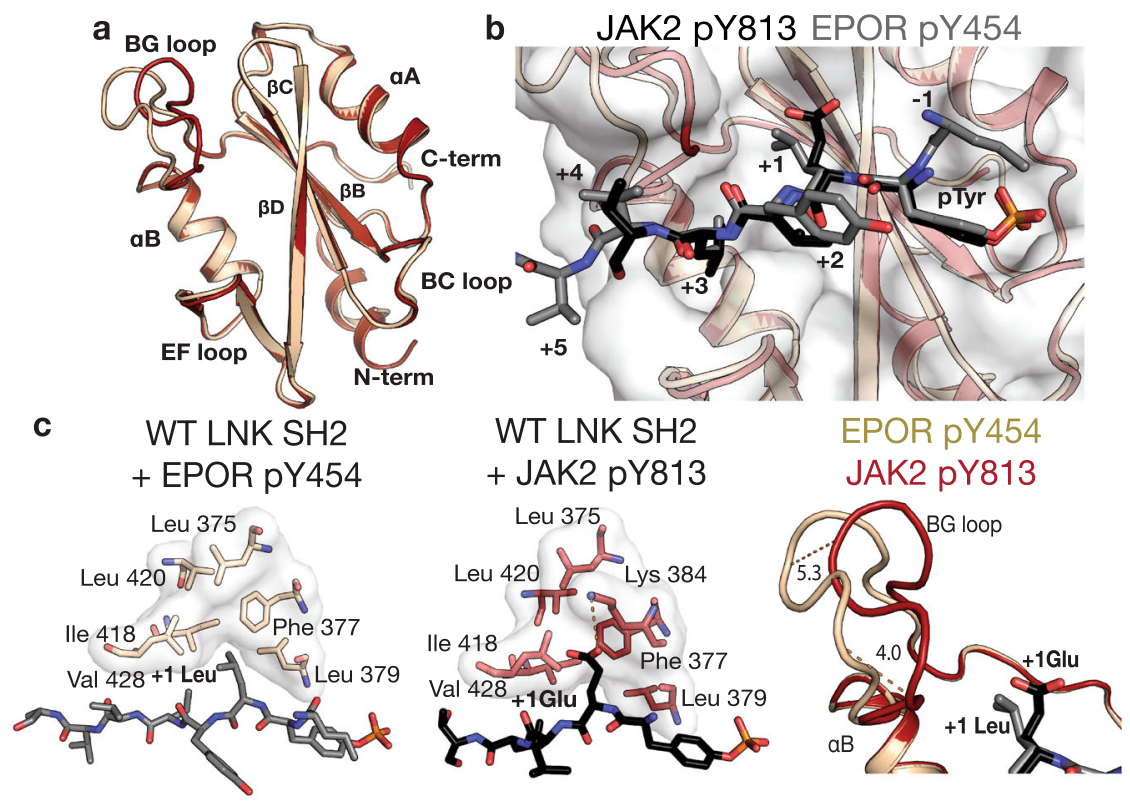

Fig. 4 Comparison of the LNK SH2 domain bound to JAK2 pY813 and EPOR pY454 phosphopeptides. a Alignment of the backbone of the WT LNK SH2 domain from the JAK2 pY813 bound structure (red) and the EPOR pY454 bound structure (purple) with secondary structural features indicated. $\mathbf{b}$ The pY813 JAK2 (black) and EPOR pY454 (white) peptides bound to the SH2 domain show a high degree of similarity. c The +1 Leu of the EPOR pY454 peptide is positioned in a hydrophobic surface on the LNK SH2 domain (left). The +1 Glu of the JAK2 pY813 peptide is positioned over the same surface, however the carboxylate also forms a salt bridge interaction with Lys 384 of LNK (middle). The LNK SH2 BG loop of the EPOR pY454 bound structure is displaced by approximately $5 \AA$ in comparison to the JAK2 pY813 bound structure (red) (right).

described in patients with a variety of diseases including the myeloproliferative neoplasms, a group of disease characterised by excessive JAK-STAT signalling resulting in hyperplasia of specific mature blood lineages ${ }^{11,13,20,38,39}$ and idiopathic erythrocytosis ${ }^{40,41}$ (Fig. 5a). We investigated three such mutations which clustered around the phosphotyrosine binding site in the LNK SH2 domain, $\mathrm{V} 402 \mathrm{M}, \mathrm{R} 415 \mathrm{C}$ and $\mathrm{R} 415 \mathrm{H}$ (V374M R387C/H in mouse respectively) (Supplementary Fig. 6).

Using mouse $\mathrm{SH} 2$ domain equivalents of these variants we investigated whether the incorporation of these mutations affected protein stability by measuring their melting point $\left(T_{\mathrm{m}}\right)$. The apo WT LNK SH2 domain had a Tm of approximately $60^{\circ} \mathrm{C}$ whereas all three mutants had a lower Tm of approximately $55^{\circ} \mathrm{C}$ (Fig. 5b). Upon addition of phosphopeptide, the melting point of the WT and both Arg 387 mutants increased, consistent with the $\mathrm{SH} 2$ domain being stabilised by the binding of peptide, whereas the V374M mutant did not. This suggests that R387C and R387H retain the ability to bind phosphorylated sequences whilst V374M does not.

In order to investigate this further, direct binding of the WT and mutant LNK SH2 domains to the JAK2 pY813 sequence was examined by SPR. All three variant LNK SH2 domains showed compromised binding to the pY813 peptide. As shown in Fig. $5 \mathrm{c}$, the $\mathrm{R} 387 \mathrm{C}$ and $\mathrm{R} 387 \mathrm{H}$ mutants displayed a 3-fold and 10-fold loss in affinity relative to the WT SH2 domain whilst V374M was too weak to quantify (Supplementary Fig. 7). Binding of the LNK $\mathrm{SH} 2$ domains was also compared to that of $\mathrm{SH} 2 \mathrm{~B}$, which has also been shown to bind JAK2 pY8132. The SH2B SH2 domain binds pY813 with an affinity of $\sim 100 \mathrm{nM}$ (Fig. $5 \mathrm{c}$ ), however the stability of the SH2B complex was significantly lower than for LNK as evidenced by much faster dissociation compared to the LNK/ phosphopeptide interaction, which is characterised by a slow offrate (Supplementary Fig. 7). Interestingly, the defect in the two R387 mutants appears to mostly be due to a compromised onrate (Supplementary Fig. 7). Together these data indicate that these three mutations surrounding the phosphotyrosine binding pocket of the LNK SH2 domain interfere with substrate binding. This is further supported by thermal shift data indicating compromised binding of phosphotyrosine (Supplementary Fig. 8).

To determine whether these mutations lead to an effect on the regulation of signalling, the three $H$. sapiens LNK SH2 domain mutants were expressed as full-length proteins in HEK293 cells and their ability to regulate signalling downstream of IFN- $\gamma$ treatment was compared to WT by luciferase assay (Fig. 5d). All three mutants had a significantly reduced capacity to regulate signalling, with the V374M mutant being the most compromised, consistent with biochemical data indicating disruption of phosphotyrosine binding.

\section{Discussion}

The data presented here reveals key determinants for LNK SH2 domain ligand recognition and identifies three high-affinity phosphotyrosine-motifs in JAK2, JAK3 and EPOR. Our structural data shows that both the JAK2 and EPOR sites bind LNK in a canonical fashion, similarly to SH2B but in contrast to APS. Although the +1 residues in the EPOR and JAK2 peptides differ, glutamate vs. leucine respectively, their binding can be accommodated by the same hydrophobic residues in the +1 pocket. Together with the structural data, our biochemical analysis indicates that residues in the $+1,+3$ (and to a lesser extent, +5 ) positions, relative to pTyr, confer specificity for the LNK SH2 domain and contribute to high-affinity binding.

In addition to the high-affinity JAK2 pY813, JAK3 pY785 and EPOR pY454 sites, we identified moderate-affinity motifs in EPOR (pY426 and pY368), FLT3 (pY591 and pY919) and C-KIT pY568 as potential binding sites for the LNK SH2 domain. These results are in agreement with previous findings $4,5,10,16,18,19$. Surprisingly, we did not find any binding sites in the TPO receptor. This may indicate that LNK interacts with the TPO signalling cascade only 
a

\begin{tabular}{cccccc}
$\begin{array}{c}\text { Human } \\
\text { mutation }\end{array}$ & $\begin{array}{c}\text { Mouse } \\
\text { equivalent }\end{array}$ & Location Disease & $\begin{array}{c}\text { JAK2 } \\
\text { status }\end{array}$ \\
\hline V402M & V374M & $\beta C$ & $\begin{array}{c}\text { ET \& } \\
\text { PMF }\end{array}$ & V617F \\
R415C & R387C & BD & IE & WT \\
R415H & R387H & BD & PV & V617F
\end{tabular}

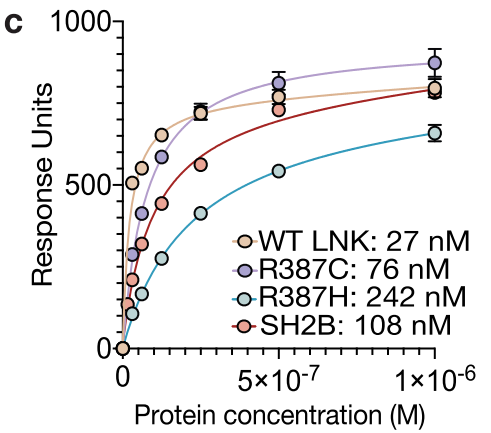

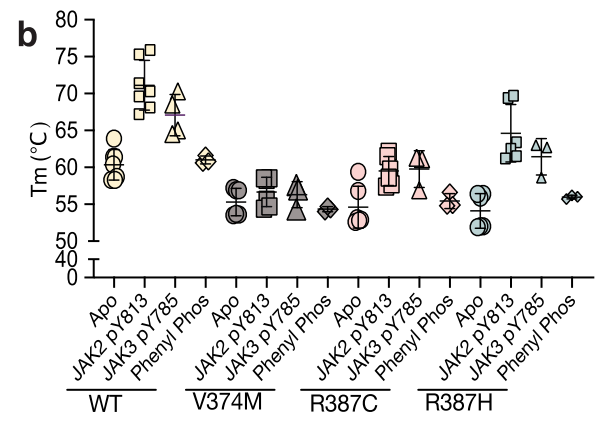

d

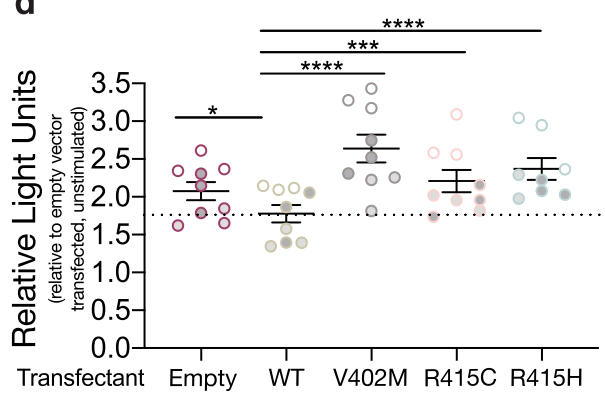

Fig. 5 Characterisation of three LNK SH2 domain mutations. a Three variants investigated in this study, their location within the LNK SH2 domain, the disease they were identified in (PV polycythemia vera, ET essential thrombocythemia, PMF primary myelofibrosis, IE idiopathic erythrocytosis) and the JAK2 status of the patient. b Melting temperature $(\mathrm{Tm})$ in ${ }^{\circ} \mathrm{C}$ for apo LNK SH2 domains and with JAK2 pY813 and JAK3 pY785 peptides and the pTyr mimetic, phenyl phosphate (Phenyl phos). Data are displayed as the mean \pm SD of technical replicates from $n=3$ independent experiments. c Fitted curves for WT, R387C and R387H LNK SH2 domain, and SH2B SH2 domain affinity for JAK2 pY813. Data are displayed as mean \pm SD from $n=5$ independent experiments for WT and R387C LNK SH2 domains and $n=4$ independent experiments for LNK R387H and $n=3$ independent experiments for SH2B SH2 domains. $K_{D}$ for each SH2 domain is indicated in nM. V374M did not bind. d Relative GAS-Firefly luciferase activity after co-transfection of HEK293 with WT, V402M (V374M), R415C (R387C) or R415H (R387H) full-length human LNK mutants and treatment with 50ng/ml rhlFN- $\gamma$. Data are displayed as mean \pm SEM from $n=3$ independent experiments where data were pooled from three experiments with each data point representing the mean of triplicate replicates of a single transfection normalised to the mean RLU (relative light units) of unstimulated empty vector control in each experiment. Statistical analysis was performed using two-sided pairwise multiple comparison with Tukey's adjustment under estimated marginal means (emmeans) function based on linear mixed-effect models using each individual experiment as a block. Significance is indicated with asterisks: ${ }^{\star} p<0.05,{ }^{\star \star \star} p<0.001,{ }^{\star \star \star \star} p<0.0001$ (empty control $p=0.05, \mathrm{~V} 402 \mathrm{M} p$ $<0.0001, \mathrm{R} 415 \mathrm{C} p=0.0006$ and R415H $p<0.001$ ).

via JAK2, although it is possible that other domains in the protein may modulate an interaction with this receptor. To date, we have been unable to purify the full-length protein.

The documentation of LNK mutations in patients with MPNs has generated interest in understanding the role of LNK in haematological diseases. Although mutations in LNK are not commonly found as drivers of MPNs, evidence has emerged that there is an overrepresentation of LNK mutations in blast-phase MPN, suggesting a role in leukemic transformation ${ }^{13,14}$. Here we investigated three single amino acid substitutions located within the LNK SH2 domain that had been identified in MPNs or the closely related disease, idiopathic erythrocytosis; V402M, R415C, and R415H (V374 and R397 in mouse) ${ }^{11,15,39,40,42}$. Of these, $\mathrm{V} 374 \mathrm{M}$ had the most severe effect on peptide binding. V374 is positioned directly underneath the pTyr ring, and pTyr binding is presumably blocked by the larger methionine side chain. The effect of the $\mathrm{R} 387 \mathrm{C} / \mathrm{H}$ LNK SH2 domain variants were more subtle. We observed a slight loss in thermal stability and affinity with these two mutants but they were still able to bind phosphorylated motifs to some extent. We hypothesise that the loss of the positive charge leads to a slight destabilisation of the BC loop (which forms the base of the pTyr binding pocket) due to disruption of an R369-E372-R387 hydrogen-bonding network. Disruption of the Arg387 could manifest as both a slightly reduced thermal stability and also a slower on-rate (the domain is not "primed" to bind pTyr). These mutations also effected LNK function in cells as in vitro analyses performed with the fulllength human protein revealed that all three mutants had a reduced capacity to negatively regulate cytokine signalling, with V402M being the most significantly compromised. These findings emphasise differences in mutation type, and highlight how identification of particular mutations in patients may be useful in assessing risk of transformation.

Taken together, these results provide a detailed picture of how the LNK SH2 domain interacts with its ligands and coupled with future studies to determine the full repertoire of LNK-interactors in cells, will allow an understanding of how defects in LNK contribute to myeloproliferative, and other haematological diseases.

\section{Methods}

Expression and purification of LNK and SH2B SH2 domains. DNA encoding the mouse LNK SH2 domain (residues 324-446) and an N-terminal NusA ${ }^{43}$ fusion separated by a TEV cleavage site was cloned (primers in Supplementary Table 2) into an pET-50b(+) vector using BamH1 and Not1, and transformed into tuner (DE3) (Novagen), BL21(DE3), or C43 E. coli cells and expression was induced by addition of $1 \mathrm{mM}$ IPTG at $18^{\circ} \mathrm{C}$ overnight. Cells were collected by centrifugation and frozen at $-30^{\circ} \mathrm{C}$. Cells from $1 \mathrm{~L}$ culture were resuspended in $40 \mathrm{~mL}$ lysis buffer (20 mM Tris ( $\mathrm{pH} 8.0$ ), $10 \mathrm{mM}$ imidazole ( $\mathrm{pH} 8.0$ ), $300 \mathrm{mM} \mathrm{NaCl}, 2 \mathrm{mM}$ TCEP, $5 \mathrm{mM}$ phenyl phosphate, $1 \mathrm{U}$ DNAse, $1 \mathrm{mM}$ PMSF, and $20 \mathrm{mg}$ lysozyme) containing EDTA-free protease inhibitor cocktail (SigmaAldrich) and lysed by sonication. Lysate was clarified by spinning cells for $10 \mathrm{~min}$ at $20,000 \times g$ before loading supernatant onto complete ${ }^{\text {sx }}$ His-Tag purification resin (Merck). Bound proteins were washed with $20 \mathrm{mM}$ tris $(\mathrm{pH} 8.0), 10 \mathrm{mM}$ imidazole $(\mathrm{pH} 8.0)$ and $300 \mathrm{mM}$ $\mathrm{NaCl}, 5 \mathrm{mM}$ phenyl phosphate followed by $20 \mathrm{mM}$ tris ( $\mathrm{pH} 8.0$ ), $30 \mathrm{mM}$ imidazole (pH 8.0) and $300 \mathrm{mM} \mathrm{NaCl}, 2 \mathrm{mM}$ TCEP $5 \mathrm{mM}$ Phenyl Phosphate. Protein was eluted in $20 \mathrm{mM}$ tris (pH 8.0), $250 \mathrm{mM}$ imidazole (pH 8.0) and $300 \mathrm{mM} \mathrm{NaCl}, 5$ $\mathrm{mM}$ Phenyl Phosphate and $2 \mathrm{mM}$ TCEP. Eluate was then cleaved with TEV protease overnight at $4{ }^{\circ} \mathrm{C}$ and subsequently purified further by size exclusion 
chromatography (Superdex 200 26/600 from GE healthcare) in TBS, 2 mM TCEP and $5 \mathrm{mM}$ Phenyl Phosphate.

DNA encoding the mouse SH2B SH2 domain and an N-terminal GST fusion separated by a TEV cleavage site was transformed into BL21(DE3) E. coli cells and expression was induced by addition of $1 \mathrm{mM}$ IPTG at $18^{\circ} \mathrm{C}$ overnight. Cells from 1 $\mathrm{L}$ culture were resuspended in $40 \mathrm{~mL}$ lysis buffer (Phosphate buffered saline (PBS), $5 \mathrm{mM}$ DTT, 1 U DNAse, $1 \mathrm{mM}$ PMSF, and $20 \mathrm{mg}$ lysozyme) and lysed by sonication. Lysate was clarified by spinning cells for $10 \mathrm{~min}$ at $20,000 \times g$ before loading supernatant onto Glutathione agarose resin purification resin (UBPbio). Bound proteins were washed with PBS, $5 \mathrm{mM}$ DTT. Protein was then cleaved with TEV protease overnight at $4{ }^{\circ} \mathrm{C}$ and cleaved SH2B SH2 domain was subsequently purified further by size exclusion chromatography (Superdex 200 10/300 from GE healthcare) in TBS, $5 \mathrm{mM} \mathrm{DTT}$.

Thermostability assays. For peptide and phenyl phosphate experiments, proteins were desalted into $100 \mathrm{mM} \mathrm{NaCl}, 20 \mathrm{mM}$ Tris ( $\mathrm{pH} 8.0$ ), $2 \mathrm{mM}$ TCEP buffer and diluted to $100 \mu \mathrm{M}$. Where peptides were used, a five-fold molar excess of peptide was added to each sample, in phenyl phosphate conditions concentration was 8 $\mathrm{mM}$. For experiments were phosphotyrosine, proteins were diluted to $30 \mu \mathrm{M}$ in PBS and in phosphate conditions, concentration was $10 \mathrm{mM}$. Ten mcroliter of each sample was transferred into a capillary and measured from 35 to $95^{\circ} \mathrm{C}$ using a Tycho N6T (Nanotemper). Data were analyzed in Prism.

SPR competition assays. SPR competition assays were performed on either a Biacore 4000 or 200 (GE Healthcare) in $10 \mathrm{mM}$ HEPES (pH 7.4), $150 \mathrm{mM} \mathrm{NaCl}$, $3.4 \mathrm{mM}$ EDTA, $0.005 \%$ Tween 20 using a streptavidin coated chip and were regenerated in $50 \mathrm{mM} \mathrm{NaOH}, 1 \mathrm{M} \mathrm{NaCl}$. A biotinylated peptide representing the IL6ST pY757 sequence was immobilised to the chip by passing over $1 \mu \mathrm{g} / \mathrm{mL}$ of peptide dissolved in $10 \mathrm{mM}$ HEPES pH 7.4, $150 \mathrm{mM} \mathrm{NaCl}, 3.4 \mathrm{mM}$ EDA, $0.005 \%$ Tween 20. 0.1-0.5 $\mu \mathrm{M}$ of WT LNK SH2 domain was pre-incubated with $2,1,0.5$, $0.25,0.125$ or $0 \mathrm{mM}$ phosphopeptides before being flowed over the chip for $240-720$ seconds at $10-30 \mu \mathrm{L} / \mathrm{min}$. Data was analysed using Prism, the response of LNK in the presence of the peptide was normalised to an LNK only control and then fitted as an $\mathrm{IC}_{50}$ curve via non-linear regression.

SPR direct binding assay. Direct binding experiments were performed on either a Biacore 4000 or 8000 (GE Healthcare) in $10 \mathrm{mM}$ HEPES (pH 7.4), $150 \mathrm{mM} \mathrm{NaCl}$, $3.4 \mathrm{mM}$ EDA, $0.005 \%$ Tween 20 using a streptavidin coated chip and were regenerated in $50 \mathrm{mM} \mathrm{NaOH}, 1 \mathrm{M} \mathrm{NaCl}$. LNK and SH2B SH2 domains were flowed over a streptavidin coated chip for $420 \mathrm{~s}$ at $30 \mu \mathrm{L} / \mathrm{min}$ with immobilised biotinylated JAK2 pY813 bound to determine binding kinetics and affinity. A reference flow cell was included by passing buffer without protein over a single lane and the sensorgrams from the reference cell were subtracted from the experimental flow cell analyses. Data were subsequently plotted in Prism.

Crystallography. All LNK constructs were buffer exchanged into low salt buffer (20 mM Tris (pH 8.0), $2 \mathrm{mM}$ TCEP and $100 \mathrm{mM} \mathrm{NaCl}$ ) and crystal trays were set up with $5 \mathrm{mg} / \mathrm{mL}$ of protein and a 2 -fold molar excess of peptide using vapour diffusion sitting drop experiments at the collaborative crystallisation centre, CSIRO (JAK2 pY813) or hanging drops set up in house (EPOR pY454). The WT LNK SH2 domain with the JAK2 pY813 peptide crystalized in $0.2 \mathrm{M}$ ammonium chloride, $20 \%$ w/v PEG 3350. The WT LNK SH2 domain with the EPOR pY454 peptide crystalised in $20 \% \mathrm{w} / \mathrm{v}$ PEG 8000, 0.05 magnesium acetate, $0.1 \mathrm{M}$ Tris (pH 8.5). All crystals were cryo-protected in paratone and immediately snap frozen in liquid nitrogen. Data was collected at the MX2 beamline at the Australian Synchrotron. Data reduction, scaling and integration was performed using $\mathrm{XDS}^{44}$. Crystal structures of the LNK SH2 domain were solved by molecular replacement (search model PDB ID: 2HDX for WT LNK SH2/JAK2 pY813 structure, and WT LNK SH2 for WT LNK SH2/EPOR pY454) using Phaser as implemented in PHENIX ${ }^{45}$. All structurers were refined using PHENIX and model building was performed in $\mathrm{COOT}^{46}$. Structures were visualised using $\mathrm{Pymol}^{47}$.

Luciferase assay. V402M, R415C and R415H mutations were introduced into the open reading frame (ORF) of pReceiver-M12-SH2B3 (Genecopoeia, Rockville, MD, USA) construct by site-directed mutagenesis using PfuTurbo DNA polymerase (Santa Clara, CA, USA) and 5\% DMSO to resolve secondary structure due to GCrich sequence in SH2B3 ORF (primers in Supplementary Table 2).

Twenty-four-well plate cultures of HEK293 cells at $70 \%$ confluency in were transfected with $100 \mathrm{ng}$ of either empty vector or full-length human LNK encoding pReceiver-M12 constructs, $145 \mathrm{ng}$ of GAS-Firefly luciferase reporter construct (a gift from Vicki Athanasopoulos) and $5 \mathrm{ng}$ of pRL-Renilla luciferase control construct using Lipofectamine 2000 transfection reagent (Invitrogen, Carlsbad, CA, USA). Twenty-four hours of post-transfection, HEK293 cells were stimulated with $50 \mathrm{ng} / \mathrm{mL}$ recombinant human IFN- $\gamma$ (Peprotech, Rock Hill, NJ, USA) for $24 \mathrm{~h}$. Firefly and Renilla luciferase activity were measured using a Luc-Pair DuoLuciferase Assay Kit (Genecopoeia, Rockville, MD, USA) and a VICTOR Nivo multimode plate reader (PerkinElmer, Waltham, MA, USA). Relative light unit (RLU) was calculated as the mean of ratios between the GAS-Firefly luciferase luminescence and Renilla luciferase luminescence, and normalised to the mean
RLU of the unstimulated empty vector-transfected samples to calculate relative GAS activity. Statistical analysis was performed using the lmerTest package in R.

Reporting summary. Further information on research design is available in the Nature Research Reporting Summary linked to this article.

\section{Data availability}

The data that support this study are available from the corresponding author upon reasonable request. Atomic coordinates for WT LNK SH2 domain with the JAK2 pY813 and EPOR pY454 phosphopeptides have been deposited in the Protein Data Bank with the accession numbers PDB 7R8W and PDB 7R8X, respectively. Source data are provided with this paper.

Received: 2 July 2021; Accepted: 30 September 2021; Published online: 20 October 2021

\section{References}

1. Huang, X., Li, Y., Tanaka, K., Moore, K. G. \& Hayashi, J. I. Cloning and characterization of Lnk, a signal transduction protein that links T-cell receptor activation signal to phospholipase C gamma 1, Grb2, and phosphatidylinositol 3-kinase. Proc. Natl Acad. Sci. 92, 11618-11622 (1995).

2. Hu, J. \& Hubbard, S. R. Structural basis for phosphotyrosine recognition by the Src homology-2 domains of the adapter proteins SH2-B and APS. J. Mol. Biol. 361, 69-79 (2006)

3. Velazquez, L. et al. Cytokine signaling and hematopoietic homeostasis are disrupted in Lnk-deficient mice. J. Exp. Med. 195, 1599-1611 (2002).

4. Tong, W. \& Lodish, H. F. Lnk inhibits Tpo-mpl signaling and Tpo-mediated megakaryocytopoiesis. J. Exp. Med. 200, 569-580 (2004).

5. Tong, W., Zhang, J. \& Lodish, H. F. Lnk inhibits erythropoiesis and Epodependent JAK2 activation and downstream signaling pathways. Blood 105, 4604-4612 (2005)

6. Bersenev, A., Wu, C., Balcerek, J. \& Tong, W. Lnk controls mouse hematopoietic stem cell self-renewal and quiescence through direct interactions with JAK2. J. Clin. Investig. 118, 2832 (2008).

7. Seita, J. et al. Lnk negatively regulates self-renewal of hematopoietic stem cells by modifying thrombopoietin-mediated signal transduction. Proc. Natl Acad. Sci. 104, 2349-2354 (2007).

8. Morris, D. L., Cho, K. W., Zhou, Y. \& Rui, L. SH2B1 enhances insulin sensitivity by both stimulating the insulin receptor and inhibiting tyrosine dephosphorylation of insulin receptor substrate proteins. Diabetes $\mathbf{5 8}$, 2039-2047 (2009).

9. Desbuquois, B., Carré, N. \& Burnol, A. F. Regulation of insulin and type 1 insulin-like growth factor signaling and action by the Grb10/14 and SH2B1/B2 adaptor proteins. FEBS J. 280, 794-816 (2013).

10. Cheng, Y. et al. LNK/SH2B3 regulates IL-7 receptor signaling in normal and malignant B-progenitors. J. Clin. Investig. 126, 1267-1281 (2016).

11. Oh, S. T. et al. Novel mutations in the inhibitory adaptor protein LNK drive JAK-STAT signaling in patients with myeloproliferative neoplasms. Blood 116, 988-992 (2010).

12. Shahin, O. A., Chifotides, H. T., Bose, P., Masarova, L. \& Verstovsek, S. Accelerated phase of myeloproliferative neoplasms. Acta Haematol. 144, 1-16 (2021).

13. Pardanani, A. et al. LNK mutation studies in blast-phase myeloproliferative neoplasms, and in chronic-phase disease with TET2, IDH, JAK2 or MPL mutations. Leukemia 24, 1713 (2010)

14. Lasho, T. L. et al. Targeted next-generation sequencing in blast phase myeloproliferative neoplasms. Blood Adv. 2, 370-380 (2018).

15. Hurtado, C. et al. LNK can also be mutated outside $\mathrm{PH}$ and $\mathrm{SH} 2$ domains in myeloproliferative neoplasms with and without V617FJAK2 mutation. Leuk. Res. 35, 1537-1539 (2011)

16. Gueller, S. et al. Adaptor protein Lnk associates with Tyr568 in c-Kit. Biochem. J. 415, 241-245 (2008).

17. Lin, D.-C. et al. Adaptor protein Lnk binds to and inhibits normal and leukemic FLT3. Blood 120, 3310-3317 (2012).

18. Gueller, S. et al. Adaptor protein Lnk inhibits c-Fms-mediated macrophage function. J. Leukoc. Biol. 88, 699-706 (2010).

19. Gueller, S. et al. Adaptor protein Lnk binds to PDGF receptor and inhibits PDGF-dependent signaling. Exp. Hematol. 39, 591-600 (2011).

20. Oh, S. T. et al. Identification of novel LNK mutations in patients with chronic myeloproliferative neoplasms and related disorders. Blood 116, 315 (2010).

21. Wang, W. et al. LNK/SH2B3 loss of function promotes atherosclerosis and thrombosis. Circ. Res. 119, e91-e103 (2016). 
22. Lavrikova, E. Y. et al. The carriage of the type 1 diabetes-associated R262W variant of human LNK correlates with increased proliferation of peripheral blood monocytes in diabetic patients. Pediatr. Diabetes 12, 127-132 (2011).

23. Hong, L. et al. Role of SH2B3 R262W gene polymorphism and risk of coronary heart disease: a PRISMA-compliant meta-analysis. Medicine 97, e13436 (2018).

24. Chaouali, M., Fernandes, V., Ghazouani, E., Pereira, L. \& Kochkar, R. Association of STAT4, TGF $\beta 1$, SH2B3 and PTPN22 polymorphisms with autoimmune hepatitis. Exp. Mol. Pathol. 105, 279-284 (2018).

25. Cai, S. et al. SH2B3, transcribed by STAT1, promotes glioblastoma progression through transducing IL-6/gp130 signaling to activate STAT3 signaling. Front. Cell Dev. Biol. 9, 274 (2021).

26. Hu, J., Liu, J., Ghirlando, R., Saltiel, A. R. \& Hubbard, S. R. Structural basis for recruitment of the adaptor protein APS to the activated insulin receptor. Mol. Cell 12, 1379-1389 (2003).

27. Hu, J. \& Hubbard, S. R. Structural characterization of a novel Cbl phosphotyrosine recognition motif in the APS family of adapter proteins. $J$. Biol. Chem. 280, 18943-18949 (2005).

28. Machida, K. et al. High-throughput phosphotyrosine profiling using $\mathrm{SH} 2$ domains. Mol. Cell 26, 899-915 (2007).

29. Babon, J. J. et al. The structure of SOCS3 reveals the basis of the extended SH2 domain function and identifies an unstructured insertion that regulates stability. Mol. Cell 22, 205-216 (2006).

30. Chen, X. et al. Crystal structure of a tyrosine phosphorylated STAT-1 dimer bound to DNA. Cell 93, 827-839 (1998).

31. Bradshaw, J. M. \& Waksman, G. Calorimetric examination of high-affinity Src SH2 domain-tyrosyl phosphopeptide binding: dissection of the phosphopeptide sequence specificity and coupling energetics. Biochemistry 38, 5147-5154 (1999).

32. Koren-Michowitz, M. et al. Adaptor protein LNK binds to and is phosphorylated by JAK3 and may serve as a scaffold for JAK3 autophosphorylation in the absence of an appropriate cytokine receptor. Blood 116, 2785 (2010).

33. Machida, K. \& Mayer, B. J. The $\mathrm{SH} 2$ domain: versatile signaling module and pharmaceutical target. Biochim. Biophys. Acta 1747, 1-25 (2005).

34. Holm, L. \& Sander, C. Dali: a network tool for protein structure comparison. Trends Biochem. Sci. 20, 478-480 (1995).

35. Perez-Garcia, A. et al. Genetic loss of SH2B3 in acute lymphoblastic leukemia. Blood 122, 2425-2432 (2013).

36. Alcina, A. et al. The autoimmune disease-associated KIF5A, CD226 and $\mathrm{SH} 2 \mathrm{~B} 3$ gene variants confer susceptibility for multiple sclerosis. Genes Immun. 11, 439-445 (2010).

37. Dale, B. L. \& Madhur, M. S. Linking inflammation and hypertension via LNK/ SH2B3. Curr. Opin. Nephrol. Hypertens. 25, 87 (2016).

38. Barbui, T. et al. The 2016 WHO classification and diagnostic criteria for myeloproliferative neoplasms: document summary and in-depth discussion. Blood Cancer J. 8, 1-11 (2018).

39. Chen, Y. et al. The polymorphisms in LNK gene correlated to the clinical type of myeloproliferative neoplasms. PLoS ONE 11, e0154183 (2016).

40. McMullin, M. F., Wu, C., Percy, M. J. \& Tong, W. A nonsynonymous LNK polymorphism associated with idiopathic erythrocytosis. Am. J. Hematol. 86, 962-964 (2011).

41. Spolverini, A. et al. Infrequent occurrence of mutations in the $\mathrm{PH}$ domain of LNK in patients with JAK2 mutation-negative 'idiopathic'erythrocytosis. Haematologica 98, e101-e102 (2013).

42. Camps, C. et al. Gene panel sequencing improves the diagnostic work-up of patients with idiopathic erythrocytosis and identifies new mutations. Haematologica 101, 1306-1318 (2016).

43. De Marco, A. Two-step metal affinity purification of double-tagged (NusA-His 6) fusion proteins. Nat. Protoc. 1, 1538 (2006).

44. Kabsch, W. Xds. Acta Crystallogr. Sect. D66, 125-132 (2010).

45. Adams, P. D. et al. PHENIX: a comprehensive Python-based system for macromolecular structure solution. Acta Crystallogr. Sect. D 66, 213-221 (2010).
46. Emsley, P. \& Cowtan, K. Coot: model-building tools for molecular graphics. Acta Crystallogr. Sect. D 60, 2126-2132 (2004).

47. DeLano, W. L. Pymol: an open-source molecular graphics tool. CCP4 Newsl. Protein Crystallogr. 40, 82-92 (2002).

48. McPhillips, T. M. et al. Blu-ice and the distributed control system: software for data acquisition and instrument control at macromolecular crystallography beamlines. J. Synchrotron Radiat. 9, 401-406 (2002).

\section{Acknowledgements}

We sincerely thank the late Prof. Tony Pawson, discoverer of the SH2 domain, who first approached and inspired us to study this protein. We are very grateful to Kazuya Machida for the details of their lnk construct. This work was supported by the National Health and Medical Research Council (NHMRC) Australia (Project grant no. 1122999, Program grant no. 1113577), an NHMRC IRIISS Grant 9000220, and a Victorian State Government Operational Infrastructure Scheme grant. J.J.B. is supported by an NHMRC fellowship. RM was supported by an Australian Postgraduate Award. This research was undertaken in part using the MX2 beamline at the Australian Synchrotron, part of ANSTO, and made use of the Australian Cancer Research Foundation (ACRF) Eiger detector ${ }^{48}$. Crystallization trials were performed at CSIRO collaborative crystallization centre (C3)

\section{Author contributions}

R.M., Y.Z., J.M.M., A.L. Carried out experiments. R.M., Y.Z., J.M.M., A.L., J.I.E., C.G.V . N.J.K., J.J.B. designed experiments and analysed and interpreted data. R.M., N.J.K., collected and analysed crystallographic data. R.M., N.J.K., J.J.B. wrote the manuscript. R.M., Y.Z., J.I.E., J.M.M., N.J.K., and J.J.B. revised the manuscript. J.J.B. Conceived project and designed the study.

\section{Competing interests}

The authors declare no competing interests.

\section{Additional information}

Supplementary information The online version contains supplementary material available at https://doi.org/10.1038/s41467-021-26394-6.

Correspondence and requests for materials should be addressed to Nadia J. Kershaw or Jeffrey J. Babon.

Peer review information Nature Communications thanks the anonymous reviewers for their contribution to the peer review of this work. Peer reviewer reports are available.

Reprints and permission information is available at http://www.nature.com/reprints

Publisher's note Springer Nature remains neutral with regard to jurisdictional claims in published maps and institutional affiliations.

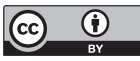

Open Access This article is licensed under a Creative Commons Attribution 4.0 International License, which permits use, sharing, adaptation, distribution and reproduction in any medium or format, as long as you give appropriate credit to the original author(s) and the source, provide a link to the Creative Commons license, and indicate if changes were made. The images or other third party material in this article are included in the article's Creative Commons license, unless indicated otherwise in a credit line to the material. If material is not included in the article's Creative Commons license and your intended use is not permitted by statutory regulation or exceeds the permitted use, you will need to obtain permission directly from the copyright holder. To view a copy of this license, visit http://creativecommons.org/ licenses/by/4.0/.

(c) The Author(s) 2021 